\title{
Coeficiente de repetibilidade para produção de frutos e seleção de matrizes de Bertholletia excelsa (Bonpl.) em castanhais nativos do estado do Acre
}

\author{
Repeatability coefficient for the fruit production and selection of Bertholletia \\ excelsa (Bonpl.) in natural stands of Acre state
}

\author{
Valéria Rigamonte Azevedo ${ }^{\mathrm{I}}$, Lúcia Helena de Oliveira Wadt ${ }^{\mathrm{II}}$, \\ Cássia Angela Pedrozo ${ }^{\text {III }}$, Fernanda Lopes da Fonseca ${ }^{\text {IV }}$, \\ Marcos Deon Vilela de Resende ${ }^{\mathrm{V}}$
}

\begin{abstract}
Resumo
Mais de 95\% da produção brasileira de castanha-da-amazônia é oriunda da coleta extrativista sendo poucos os plantios para produção comercial. Buscando contribuir com informações básicas para o melhoramento genético da castanheira, este estudo teve por objetivos: estimar os coeficientes de repetibilidade para o número de frutos (NFP); determinar o número adequado de medições para uma eficiente seleção de matrizes; e, selecionar castanheiras promissoras em dois castanhais nativos, no Estado do Acre. O estudo foi realizado em parcelas permanentes no Filipinas (Resex Chico Mendes, Epitaciolândia-AC) e Cachoeira (PAE Chico Mendes, Xapuri-AC). O número de frutos por planta (NFP) foi avaliado em 140 castanheiras no Filipinas, no período de 2002 a 2014 (13 anos), enquanto no Cachoeira foram 175 castanheiras de 2009 a 2014 (seis anos). As análises genético-estatísticas foram feitas com base na metodologia de modelos mistos, utilizando-se o software Selegen-REML/BLUP, Modelo 63. Foram selecionadas 20 matrizes, sendo 08 no Filipinas e 12 no Cachoeira. Em ambos os castanhais, a variância fenotípica permanente (Vfp) representou a maior porcentagem da variância fenotípica individual (Vf). O coeficiente de repetibilidade foi considerado moderado para o Cachoeira e alto para o Filipinas. Considerando um coeficiente de determinação de 90\%, seriam necessários sete anos de avaliação do NFP para o Cachoeira e seis anos para o Filipinas. O banco de dados existentes para a produção de frutos das castanheiras é suficiente para se realizar a seleção de matrizes com alta produção de frutos. Com base nos valores estimados espera-se que a nova população apresente uma produção média de 465,3 frutos.arv ${ }^{-1}$, indicando um ganho na produção de frutos cerca de 2,4 vezes para o Cachoeira e 4,2 para o Filipinas.
\end{abstract}

Palavras-chave: Cultivo; Melhoramento genético; Castanha-da-amazônia

\footnotetext{
Bióloga, MSc, Professora do Instituto Federal do Acre, Campus Sena Madureira, Rua Francisca Sousa da Silva, Bairro Getúlio Nunes Sampaio, 318 , CEP 69 940-000, Sena Madureira (AC), Brasil. valeria.assis@ifac.edu.br (ORCID: 0000-0002-0792-8499)

Engenheira Florestal, Dra., Pesquisadora da Embrapa Rondônia, Rodovia Br 364, km 5,5, CEP 76815-800, Porto Velho (RO), Brasil. lucia.wadt@ embrapa.br (ORCID: 0000-0002-5018-7550)

III Engenheira Agrônoma, Drª -, Pesquisadora da Embrapa Roraima, Rod. BR 174, Km 08, Distrito Industrial, CEP 69301-970, Boa Vista (RR), Brasil. cassia.pedrozo@embrapa.br(ORCID: 0000-0002-0474-263X)

IV Engenheira Florestal, Analista de P\&D da Embrapa Acre, Rod. BR 364, Km 14, CEP 69900-970, Rio Branco (AC), Brasil. fernanda.fonseca@embrapa. br (ORCID: 0000-0001-7289-6684)

Estatístico, Dr., Pesquisador da Embrapa Florestas, Estrada da Ribeira, Km 111, Bairro Guaraituba, CEP 83411-000, Colombo (PR), Brasil. marcos. deon@gmail.com (ORCID: 0000-0002-3087-3588)
} 


\begin{abstract}
More than 95\% of Brazil nut production in Brazil comes from the extractive collection, with few plantations for commercial production. To contribute with Brazil nut tree genetic improvement, this study aimed to estimate number of fruits (NFP) repeatability coefficients; determine the appropriate number of measurements for efficient selection; and select Brazil nut promising trees from two Brazil nut stands in the state of Acre. The study was carried out in permanent plots in Filipinas (Resex Chico Mendes, Epitaciolândia-AC) and Cachoeira (PAE Chico Mendes, Xapuri-AC). The number of fruits per plant (NFP) was estimated at 140 Brazil nut trees from 2002 to 2014 (13 years), while in Cachoeira there were 175 Brazil nut trees from 2009 to 2014 (six years). Genetic-statistical analyzes were performed based on the mixed model methodology, using the Selegen-REML/BLUP Model 63. Twenty matrices were selected, 08 in Filipinas and 12 in Cachoeira. In both Brazil nuts stands, the permanent phenotypic variance (Vfp) represented the largest percentage of the individual phenotypic variance (Vf). The repeatability coefficient was considered moderate for Cachoeira and high for Filipinas. Considering a $90 \%$ coefficient of determination, it would take seven years of NFP assessment for Cachoeira and six years for Filipinas. The existing database for the Brazil nut production is sufficient to make selection for matrices with high fruit production. Based on the estimated values we expect the new population to have an average yield of 465.3 fruits.arv ${ }^{-1}$, indicating a gain in fruit production of about 2.4 times for Cachoeira and 4.2 for Filipinas.
\end{abstract}

Keywords: Crop; Genetic improvement; Brazil nut

\title{
Introdução
}

Bertholletia excelsa (Bonpl.) é uma espécie arbórea nativa da região amazônica, com importância econômica para milhares de trabalhadores rurais e urbanos (RUIZ, 2008). É a única espécie do gênero e sua importância econômica está na produção de amêndoas conhecidas como castanha-do-brasil, castanha-do-pará ou castanha-da-amazônia. Ocorre em cerca de 325 milhões de hectares de florestas naturais (STOIAN, 2004), sendo que, no Brasil, é encontrada em densidades variáveis nos Estados do Acre, Amapá, Amazonas, Pará, Roraima, Rondônia, Maranhão e Mato Grosso (MÜLLER; CALVAZARA, 1986).

Mais de 95\% da produção brasileira de castanha-da-amazônia é oriunda da coleta extrativista em castanhais nativos (WADT et al., 2008) sendo poucos os castanhais plantados para produção comercial de frutos e sementes (WADT e KAINER, 2009). Essa particularidade faz com que o cultivo da castanheira visando à produção de frutos tenha uma importância significativa para a Amazônia. Apesar de existirem alguns plantios comerciais, como por exemplo o da Fazenda Aruanã, no município de Itacoatiara-AM, estes apresentam baixa produtividade de frutos (CAVALCANTE, 2008) principalmente, por problemas relacionados à incompatibilidade de matrizes aparentadas (HOMMA, 1989).

Dessa forma, o plantio comercial de B. excelsa para a produção de frutos e sementes deve ser realizado com mudas de elevada qualidade genética, proveniente de plantas-matrizes selecionadas, especialmente pelo fato da espécie ser alógama e com algum sistema de autoincompatibilidade (MORITZ, 1984; O'MALLEY et al., 1988; CAVALCANTE, 2008).

Questões técnicas relacionadas à conservação e melhoria da capacidade de germinação das sementes; da produção de mudas e da enxertia para obtenção de plantas baixas e precoces já foram resolvidas em estudos básicos desde a década de 90 (MÜLLER et al., 1995; LOCATELLI; SOUZA, 1990; MOREIRA, 1993; KAINER et al., 1999; LOCATELLI et al., 2005; NASCIMENTO et al., 2010). No entanto, os plantios comerciais não ocorreram em escala e não existe no mercado material genético registrado para a produção de mudas.

Na década de 60, Hans Müller selecionou clones de alta produtividade em castanhais nativos, sendo os principais: Santa Fé I e II, Manuel Pedro I e II, C-606, C-609, C-612, C-614, C-710 e C-722, oriundos de Alenquer e Oriximiná, estado do Pará (NASCIMENTO et al., 2010). Estes clones foram plantados no campo experimental da Embrapa Amazônia Oriental (CPATU) e posteriormente levados para a Fazenda Aruanã S.A. em Itacoatiara-AM. Até 2013, existia apenas um jardim clonal de castanheiras, o da Fazenda Aruanã S.A., uma vez que o jardim do CPATU foi destruído pelo fogo (CAVALCANTE, 2008). Em 2013, foram implantados três pomares de sementes clonais de castanheira 
nas Embrapas Acre, Roraima e Amapá, com matrizes selecionadas com base em dados de produção de frutos em parcelas permanentes da Rede Kamukaia.

Até o momento, não existem variedades e nem clones definidos e devidamente lançados para a espécie, sendo encontrado no Registro Nacional de Cultivares (RNC) do MAPA (Ministério da Agricultura, Pecuária e Abastecimento), no Brasil, apenas o registro da espécie (Registro de № 23550). No Peru, o Instituto de Investigações da Amazonia Peruana (IIAP) desenvolve trabalhos de seleção e avaliação de clones de castanheira oriundos de populações naturais desde 2000 quando iniciou com a seleção de matrizes superiores. Atualmente existem 160 matrizes selecionadas em todo o departamento de Madre de Dios. Em 2010, este grupo de pesquisadores publicou um manual de cultivo, com informações e técnicas avançadas para superar alguns gargalos existentes para o sucesso do cultivo da castanheira (CORVERA-GOMRINGER et al., 2010).

A seleção em espécies perenes, especialmente para características de produção, demanda muito tempo devido aos longos ciclos produtivo e reprodutivo e à necessidade de condução de experimentos grandes e onerosos. Com o uso da metodologia de modelos mistos (REML/BLUP), é possível abreviar esse tempo por meio da seleção de matrizes produtivas em populações naturais (sem o uso de delineamento experimental), via uso de várias medições em cada planta e predição de seus valores fenotípicos permanentes (RESENDE, 2002) obtidos pela média das medidas repetidas, ponderadas pelo coeficiente de repetibilidade temporal da característica. Este é definido como a correlação entre as medidas de determinado caráter em um mesmo indivíduo por meio de avaliações repetidas no tempo, sem a necessidade de utilizar progênies (RESENDE, 2002). Além de medir a capacidade que os organismos têm de repetir a expressão do caráter, o coeficiente de repetibilidade também permite estimar o número de medições necessárias para determinado caráter, proporcionando maior eficiência na seleção de genótipos (FALCONER, 1987). Resende (2002) afirma que a metodologia dos modelos mistos (REML/BLUP) em nível individual é a melhor alternativa para seleção de plantas perenes.

Neste sentido, o coeficiente de repetibilidade tem sido utilizado em espécies perenes para melhorar a eficiência de seleção, como por exemplo em: coqueiro - Cocos nucifera (SIQUEIRA, 1982), seringueira - Hevea brasilienses (GONÇALVES et al., 1982; VASCONCELOS et al., 1985), cajueiro Anacardium occidentale (CAVALCANTI et al., 1999), aceroleira - Malpighia emarginata (LOPES et al., 2001), pupunha - Bactris gasipaes (FARIAS-NETO et al, 2002), araçazeiro - Psidium cattleyanum e pitangueira - Eugenia uniflora (DANNER et al, 2010).

No caso da castanheira, a ausência de critérios para seleção de material genético adequado para o plantio pode gerar tanto a perda de produtividade como a perda na qualidade dos frutos. Além disso, a seleção de matrizes baseada apenas em procedimento fenotípico pode ser ineficiente por confundir os efeitos genotípicos e ambientais.

Buscando contribuir com informações básicas para o melhoramento genético de Bertholletia excelsa, o presente trabalho teve por objetivos: 1) estimar os coeficientes de repetibilidade para o número de frutos da castanheira, em dois castanhais nativos no Estado do Acre; 2) determinar o número adequado de medições necessárias para uma eficiente seleção nestes dois castanhais; e 3) selecionar árvores promissoras de B. excelsa em cada castanhal.

\section{Material e métodos}

O estudo foi realizado em dois castanhais nativos, nos quais existem parcelas permanentes para o monitoramento da produção anual de frutos de castanheira (WADT et al., 2008). Um deles está localizado na Reserva Extrativista Chico Mendes, Seringal Filipinas (Coordenadas UTM 19L 536675; 8806950) e o outro no Projeto de Assentamento Extrativista Chico Mendes, conhecido como Seringal Cachoeira (Coordenadas UTM 19L 0566157; 8803448), ambos no Estado do Acre.

A densidade de castanheiras e a produtividade de frutos nestes dois castanhais é diferente, sendo que, no Cachoeira, a densidade é 1,6 vezes maior que no Filipinas e a produtividade (frutos. ha $^{-1}$ ) 2,4 vezes maior (WADT et al., 2008).

O clima da região é quente e úmido e a temperatura média anual $26^{\circ} \mathrm{C}$. A precipitação 
média anual está em torno de $2.000 \mathrm{~mm}$ (ACRE, 2006). No Cachoeira, a floresta é ombrófila com os seguintes tipos: (i) floresta aberta dominada por bambus (26,6\%); (ii) floresta de bambus mesclada com florestas com árvores emergentes (38\%); (iii) floresta densa com árvores emergentes mesclada com floresta aberta dominada por bambus (9\%); (iv) floresta aberta com árvores emergentes (14,5\%); e (v) floresta aberta de várzea com palmeiras (2\%) (COOPEAGRO, 2001). Na RESEX Chico Mendes, região do Filipinas, $27 \%$ da vegetação é composta por florestas densas e o restante (73\%) por florestas abertas (ACRE, 2006).

O número de frutos por planta (NFP), obtido pela contagem de frutos caídos sob a copa de cada árvore foi avaliado em 140 castanheiras no Filipinas, no período de 2002 a 2014 (treze anos), enquanto que, no Cachoeira foram avaliadas 175 castanheiras de 2009 a 2014 (seis anos). O conjunto de dados foi obtido no banco de dados do projeto Kamukaia (liderado pela Embrapa Acre) e utilizado nas análises genético-estatísticas.

As análises genético-estatísticas foram feitas com base na metodologia de modelos mistos, utilizando-se o software Selegen-REML/BLUP, Modelo 63. Este modelo é indicado em casos em que são tomados dados repetidos em plantas individuais, sem o uso de delineamentos experimentais (RESENDE, 2006).

O modelo utilizado consistiu em:

$$
y=X_{m}+W_{p}+\mathrm{e}
$$

Em que: $y=$ vetor de dados; $m$ = vetor dos efeitos de medição, assumidos como fixos, somados à média geral; $p=$ vetor dos efeitos permanentes de plantas (efeitos genotípicos + efeitos de ambiente permanente), assumidos como aleatórios; $e=$ vetor de erros ou resíduos. As letras X e W representam as matrizes de incidência para os referidos efeitos (RESENDE, 2002).

Os componentes da variância fenotípica fornecidos pelo modelo foram os seguintes:

$\mathrm{V}_{\mathrm{fp}}$ : variância fenotípica permanente entre plantas (genotípica + ambiental permanente de um ano para outro);

$\mathrm{V}_{\mathrm{et}}$ : variância ambiental temporária;

$\mathrm{V}_{\mathrm{f}}$ : variância fenotípica individual;

r: repetibilidade individual;

$\mathrm{r}_{\mathrm{m}}$ : repetibilidade da média de $m$ anos de avaliação;

$\mathrm{A}_{\mathrm{cm}}$ : acurácia da seleção baseada na média de $m$ anos de avaliação;

\section{Resultados e discussão}

Os valores médios de produção de frutos (valores fenotípicos) obtidos durante os períodos de monitoramento foram de 188,23 frutos.árv ${ }^{-1}$ e 95,34 frutos.árv ${ }^{-1}$, respectivamente, para o Cachoeira e o Filipinas (Tabela 1). Provavelmente essa diferença entre castanhais pode ser devido à estrutura genética e às condições ambientais de cada um.

Em ambos os castanhais, a variância fenotípica permanente $\left(\mathrm{V}_{\mathrm{fp}}\right)$ foi maior que a variância ambiental temporária $\left(\mathrm{V}_{\mathrm{et}}\right)$ (Tabela 1), indicando que a variação ambiental, para a característica avaliada (NFP), foi relativamente baixa quando comparada a variação fenotípica permanente existente entre as plantas. Em dois castanhais naturais avaliados em Roraima (PEDROZO et al., 2015), foram obtidos valores de $\mathrm{V}_{\mathrm{et}}$ superiores a valores de $\mathrm{V}_{\mathrm{fp}}$, indicando para aquelas populações que a variação ambiental foi maior que as variações genéticas. Sujii et al. (2015), estudando genética de populações de Bertholletia excelsa em nove castanhais naturais representando cinco regiões da Amazônia, verificaram que os castanhais do Acre (região A) foram os mais divergentes com relação às outras regiões. Os castanhais do Acre e de Roraima (Região E) apresentaram cerca de $35 \%$ de divergência genética (distância genética de Nei); e, além disso, as duas populações estudadas em Roraima foram idênticas geneticamente (distância genética de Nei) enquanto no Acre houve diferenças genéticas entre as populações estudadas. Provavelmente, as diferenças obtidas entre $V_{f p}$ e $V_{\text {et }}$ neste estudo e no 
de Pedrozo et al. (2015) estão relacionadas à estrutura genética das populações, bem como aos efeitos ambientais bióticos e abióticos que atuam sobre os mesmos.

Resultados similares de variação fenotípica superior à variação ambiental foram observados em outras espécies perenes para características de frutos como pessegueiro (Prunus persica L. Batsch), laranjeiras-doces (Citrus sinensis (L.) Osbeck), mangueira (Mangifera indica L.) e aceroleira (Malpighia emarginata) (LOPES et al., 2001; COSTA, 2003; NEGREIROS et al., 2008; BRUNA et al, 2012). Esse tipo de resultado é importante, pois indica a possibilidade de se obter sucesso na seleção de genótipos.

O coeficiente de repetibilidade, ou seja, a medida da capacidade que as castanheiras têm de repetir a expressão do caráter produção ao longo do tempo, é outro parâmetro importante a ser avaliado quando se deseja selecionar genótipos superiores em populações naturais. Neste estudo, os valores de repetibilidade para ambos os castanhais foram considerados, segundo Resende (2002), moderado $(0,57)$ para o Cachoeira e alto para o Filipinas $(0,62)$ (Tabela 1$)$. No estudo de Pedrozo et al. (2015) este coeficiente foi inferior para a característica NFP $(0,33$ e 0,31 para os dois castanhais estudados) indicando que os castanhais do Acre são mais promissores para a seleção de genótipos superiores quando comparados aos de Roraima.

As estimativas de repetibilidade podem variar de acordo com a natureza da característica avaliada; com as propriedades genéticas dos genótipos; com as condições em que os indivíduos se desenvolvem; e se o genótipo em que se realizam as medidas repetidas se encontra estabilizado (CRUZ et al., 2004). Segundo Pereira et al. (2002), quando o estudo de repetibilidade é feito com genótipos ainda não estabilizados, pode-se encontrar baixo valor de repetibilidade, sem que necessariamente, a solução do problema esteja no aumento do número de repetições. As baixas estimativas de repetibilidade obtidas para os castanhais de Roraima (PEDROZO et al., 2015) podem levantar uma hipótese de que aqueles castanhais são mais novos que os do Acre, estando ainda em fase de estabilização.

$\mathrm{Na}$ ausência de estudos sobre a herdabilidade de caracteres produtivos de Bertholletia excelsa, as estimativas de repetibilidade tornam-se importantes para programas de melhoramento, pois expressam o valor máximo que a herdabilidade pode atingir (FALCONER, 1981).

\section{Tabela 1 - Componentes de variância e estimativas dos parâmetros fenotípicos para o número de frutos por planta (NFP) de Bertholletia excelsa, em dois castanhais nativos (Cachoeira e Filipinas) no Acre}

Table 1 - Components of variance and estimates of Bertholletia excelsa phenotypic parameters for the number of fruits per plant (NFP) in two Brazil nut stands (Cachoeira e Filipinas) in Acre

\begin{tabular}{lcc}
\hline & Cachoeira & Filipinas \\
\hline $\mathbf{V}_{\mathrm{fp}}$ (variância fenotípica permanente entre plantas) & $18.303,6$ & $10.607,2$ \\
$\mathbf{V}_{\mathrm{et}}$ (variância de ambiente temporário) & $13.424,3$ & $6.474,3$ \\
$\mathbf{V}_{\mathrm{f}}$ (variância fenotípica individual) & $31.727,8$ & $17.081,5$ \\
$\mathbf{r}$ (repetibilidade individual) & 0,576 & 0,620977 \\
\pm Desvio Padrão & $\pm 0,0821$ & $\pm 0,0721$ \\
$\mathbf{r}_{\mathrm{m}}$ (repetibilidade da média de anos avaliados) & 0,87 & 0,92 \\
$\mathbf{A}_{\mathrm{cm}}$ (acurácia da seleção baseada na média de anos avaliados) & 0,93 & 0,96 \\
\hline Média geral de produção de frutos & 188,23 & 95,34 \\
\hline
\end{tabular}

Fonte: Autores (2018)

A análise da repetibilidade permite também estimar o número de medições necessárias em cada indivíduo para que haja eficiência na seleção para determinado caráter. Nesse estudo, considerando um coeficiente de determinação de $90 \%$, seriam necessários sete anos de avaliação 
do NFP para o castanhal do Cachoeira e seis anos para o do Filipinas. Se a seleção for menos rígida $\left(\mathrm{C}_{2}=80 \%\right)$, três anos de avaliação do NFP são suficientes para ambos os castanhais (Tabela 2). Com base nos resultados obtidos, verifica-se que o banco de dados existentes para a produção de frutos das castanheiras é suficiente para se realizar a seleção de matrizes de castanheira com alta produção de frutos. Em Roraima, por exemplo, foram necessários, em média, nove anos de avaliação para se conseguir coeficientes de determinação maior que 80\% (PEDROZO et al., 2015).

A acurácia dos valores fenotípicos permanentes baseados em $m$ anos de avaliação $\left(\mathrm{A}_{\mathrm{cm}}\right)$ foi alta (mínimo de 0,76 e 0,79 para Cachoeira e Filipinas, respectivamente), comparado aos valores observados por Pedrozo et al. (2015), em Roraima (0,57 e 0,56). Isto indica melhor eficácia na estimativa dos valores genotípicos para os castanhais do Acre. Com relação à eficiência das $m$ avaliações quando comparadas com a situação em que apenas uma avaliação é realizada (Ef), os valores médios para o Acre foram relativamente menores que os encontrados por Pedrozo et al. (2015), 1,6 em Roraima e 1,2 para o Acre, confirmando a maior repetibilidade dos valores nos castanhais do Acre. Em suma, a produção de frutos nos castanhais de Roraima estudados por Pedrozo et al. (2015) teve um efeito ambiental temporário significativo aumentando a variabilidade dos valores ao longo dos anos, enquanto nos castanhais do Acre esse efeito ambiental foi muito menor, resultando em valores de produção de frutos menos variáveis ao longo dos anos.

\section{Tabela 2 - Coeficiente de determinação $\left(\mathrm{C}_{2}\right)$, acurácia dos valores fenotípicos permanentes} baseados em $m$ anos de avaliação $\left(A_{c m}\right)$ e eficiência das $m$ avaliações quando comparadas com a situação em que apenas uma avaliação é realizada $(\mathrm{Ef})$, para número de frutos de Bertholletia excelsa por planta (NFP), avaliado em dois castanhais nativos (Cachoeira e Filipinas) no Acre

Table 2 - Coefficient of determination $\left(\mathrm{C}_{2}\right)$, accuracy of permanent phenotypic values based on $m$-year review $\left(\mathrm{A}_{\mathrm{cm}}\right)$ of $m$ and efficiency ratings when compared to the situation where only one evaluation is performed (Ef) to the number of Brazil nut fruits per plant (NFP), valued at two

Brazil nut stands (Cachoeira and Filipinas) in Acre

\begin{tabular}{ccccccc}
\hline \multicolumn{3}{c}{ Castanhal Cachoeira } & \multicolumn{3}{c}{ Castanhal Filipinas } \\
\hline $\mathbf{M}$ & $\mathrm{C}_{2}$ & $\mathrm{~A}_{\mathrm{cm}}$ & $\mathrm{Ef}$ & $\mathrm{C}_{2}$ & $\mathrm{~A}_{\mathrm{cm}}$ & $\mathrm{Ef}$ \\
$\mathbf{1}$ & 0,576892 & 0,759534 & 1,000000 & 0,620977 & 0,788021 & 1,000000 \\
$\mathbf{2}$ & 0,731682 & 0,855384 & 1,126196 & 0,766176 & 0,87531 & 1,110776 \\
$\mathbf{3}$ & 0,803551 & 0,896410 & 1,180211 & 0,830941 & 0,91156 & 1,156771 \\
$\mathbf{4}$ & 0,845054 & 0,919268 & 1,210305 & 0,867610 & 0,931456 & 1,182019 \\
$\mathbf{5}$ & 0,872079 & 0,933852 & 1,229506 & 0,891208 & 0,944038 & 1,197986 \\
$\mathbf{6}$ & 0,891077 & 0,943969 & 1,242826 & 0,907665 & 0,952715 & 1,208997 \\
$\mathbf{7}$ & 0,905162 & 0,951400 & 1,252610 & 0,919798 & 0,959061 & 1,217050 \\
$\mathbf{8}$ & 0,916021 & 0,957090 & 1,260101 & 0,929113 & 0,963905 & 1,223197 \\
$\mathbf{9}$ & 0,924649 & 0,961587 & 1,266021 & 0,936489 & 0,967724 & 1,228043 \\
$\mathbf{1 0}$ & 0,931669 & 0,965230 & 1,270818 & 0,942475 & 0,970811 & 1,231961 \\
\hline
\end{tabular}

Fonte: Autores (2018)

A seleção das matrizes superiores para produção de frutos foi feita com base no ranqueamento do valor fenotípico permanente $(\mu+\mathrm{fp})$ e o valor mínimo de valor fenotípico (Fp) igual a 200 frutos. Com base nesses critérios foram selecionadas 20 matrizes, sendo 12 procedentes do Cachoeira e oito do Filipinas (Tabela 3), representando 6\% e 10\% de cada castanhal, respectivamente. 
$\mathrm{Na}$ Tabela 3 estão apresentados o ranqueamento das matrizes selecionadas com base no índice adaptado de Mulamba; Mock (1978); os valores fenotípicos permanentes ( $\mu+f \mathrm{f})$; o ganho de produção; e a nova média esperada para cada população após a seleção dos genótipos de Bertholletia excelsa (12 matrizes no Cachoeira e 08 matrizes no Filipinas). Para todas as matrizes, os valores fenotípicos permanentes foram maiores que os valores fenotípicos resultando em ganho na produção com a seleção. No entanto, vale ressaltar que esse ganho não é só genético, ele contém a variação permanente do ambiente, ou seja, apenas o valor da variação ambiental temporária é que foi desconsiderado.

A seleção desses 20 genótipos superiores permite a formação de uma nova população de castanheiras com valor fenotípico permanente médio esperado de 448 frutos.arv $^{-1}$ para o Cachoeira e 399,7 frutos.arv ${ }^{-1}$ para o Filipinas. Em relação às populações originais, calcula-se um ganho na produção de frutos de 2,4 vezes para o Cachoeira e 4,2 para o Filipinas. Em termos de melhoramento genético, a nova população formada com os genótipos selecionados é bastante promissora, uma vez que com três a quatro anos de avaliação da produção de frutos será possível fazer novas seleções, considerando um coeficiente de determinação de $80 \%$.

Tabela 3 - Valores fenotípicos (Fp), valores fenotípicos permanentes $(\mu+f p)$, ganho genético para a variável objeto da seleção (Ganho) e nova média do material genético após a seleção de Bertholletia excelsa nos castanhais do Cachoeira e Filipinas, para a variável número de frutos por planta (NFP)

Table 3 - Phenotypic values (Fp), permanent phenotypic values $(\mu+\mathrm{fp})$, genetic gain for the selection variable (Gain) and new mean after Bertholletia excelsa selection from Cachoeira and Filipinas, for number of fruits per plant (NFP)

\begin{tabular}{|c|c|c|c|c|c|c|c|c|c|c|}
\hline \multirow[b]{2}{*}{ Ranque } & \multicolumn{5}{|c|}{ Cachoeira } & \multicolumn{5}{|c|}{ Filipinas } \\
\hline & Árvore & $\mathbf{F p}$ & $\boldsymbol{\mu}+\mathbf{F p}$ & Ganho & $\begin{array}{l}\text { Nova } \\
\text { Média }\end{array}$ & Árvore & $\mathbf{F p}$ & $\boldsymbol{\mu}+\mathbf{F p}$ & Ganho & $\begin{array}{l}\text { Nova } \\
\text { Média }\end{array}$ \\
\hline 1 & 238 & 425,2 & 613,4 & 425,2 & 613,4 & 50 & 404,5 & 499,8 & 404,5 & 499,8 \\
\hline 2 & 131 & 274,9 & 463,0 & 350,0 & 538,2 & 231 & 339,4 & 434,7 & 371,9 & 467,3 \\
\hline 3 & 290 & 264,0 & 452,3 & 321,4 & 509,6 & 294 & 320,8 & 416,2 & 354,9 & 450,3 \\
\hline 4 & 319 & 254,9 & 443,2 & 304,7 & 493,0 & 339 & 306,3 & 401,6 & 342,7 & 438,0 \\
\hline 5 & 1 & 250,6 & 438,8 & 293,9 & 482,2 & 352 & 305,9 & 401,3 & 335,4 & 430,7 \\
\hline 6 & 204 & 246,6 & 434,8 & 286,0 & 474,3 & 2017 & 272,6 & 367,9 & 324,9 & 420,3 \\
\hline 7 & 188 & 244,8 & 433,0 & 280,1 & 468,4 & 391 & 244,6 & 339,9 & 313,5 & 408,8 \\
\hline 8 & 119 & 235,8 & 424,0 & 274,6 & 462,8 & 113 & 240,8 & 336,1 & 304,4 & 399,7 \\
\hline 9 & 55 & 235,3 & 423,9 & 270,2 & 458,5 & & & & & \\
\hline 10 & 134 & 219,6 & 407,8 & 265,1 & 453,4 & & & & & \\
\hline 11 & 199 & 217,5 & 405,7 & 260,8 & 449,1 & & & & & \\
\hline 12 & 311 & 210,1 & 398,4 & 256,6 & 444,8 & & & & & \\
\hline $\mathrm{Mg}$ & & & 188 & & & & & 95,3 & & \\
\hline Ms & & & 444 & & & & & 399,7 & & \\
\hline
\end{tabular}

Fonte: Autores (2018)

Em que: $\mathrm{Mg}$ = média geral dos valores fenotípicos; Ms = média dos valores fenotípicos permanentes dos genótipos selecionados. 


\section{Conclusão}

A avaliação de Bertholletia excelsa possibilitou estimativas de componentes de variância fenotípica e ambiental e a predição de valores fenotípicos permanentes visando à seleção de matrizes superiores para o caráter número de frutos por planta (NFP).

A variância fenotípica permanente, para NFP, entre plantas, foi maior que a variância de ambiente temporário para os dois castanhais estudados, indicando pouca influência ambiental na expressão da característica, e, portanto, boas perspectivas de sucesso na seleção de genótipos.

Os dados de produção (NFP) apresentaram coeficientes de repetibilidade (r) médio para o castanhal do Cachoeira e alto para o Filipinas.

Para uma seleção, considerando-se um coeficiente de determinação de 90\%, são necessários sete anos de avaliação do NFP para o Cachoeira e seis anos no Filipinas. Considerando um coeficiente de determinação de $80 \%$, três anos de avaliação são suficientes para ambos os castanhais.

Foram selecionadas 20 matrizes promissoras para produção de frutos, e estima-se que uma nova população formada por estes genótipos selecionados possa produzir 2,4 e 4,2 vezes a mais comparado com os castanhais originais do Cachoeira e Filipinas, respectivamente.

\section{Referências}

ACRE. Governo do Estado do Acre. Programa Estadual de Zoneamento Ecológico-Econômico do Estado do Acre. Zoneamento Ecológico-Econômico do Acre Fase II: Documento Sínteseescala 1: 250.000. Rio Branco: SEMA, 2006. 356p.

BRUNA, E. D.; MORETO, A. L.; DALBO, M. A. Repeatability in the selection of clones of peach for the south coastal region of the state of Santa Catarina, Brazil. Revista Brasileira de Fruticultura, Jaboticabal, v.34, n.1, p.206-215, 2012.

CAVALCANTE, M. C. Visitantes florais e polinização da castanha-do-brasil (Bertholletia excelsa H.\&B.) em cultivo na Amazônia central. 2008. 77p. Dissertação (Mestrado em Zootecnia) - Universidade Federal do Ceará, Fortaleza, 2008.

CAVALCANTI, J. J. V. et al. Repetibilidade e número de avaliações necessárias à seleção de clones de cajueiro-anão-precoce. Fortaleza: Embrapa-CNPAT, 1999. 12p. (Boletim de Pesquisa, 23).

COSTA, J. G. Estimativas de repetibilidade de alguns caracteres de produção em mangueira. Ciência Rural, Santa Maria, vol.33, n.2, pp. 263-266, 2003.

COOPEAGRO. Plano de Desenvolvimento Sustentável do Projeto de Assentamento Chico Mendes, Município de Epitaciolândia, Estado do Acre. Epitaciolândia, Acre: Coopeagro, 2001.

CORVERA-GOMRINGER, R. et al. La castaña amazónica (Bertholletia excelsa): Manual de cultivo. IIAP, Madre de Dios. 71p., 2010.

CRUZ, C. D.; REGAZZI, A. J.; CARNEIRO, P. C. S. Modelos biométricos aplicados ao melhoramento genético. Viçosa: UFV, 2004. 480 p.

DANNER, M. A. et al. Repetibilidade de caracteres de fruto em araçazeiro e pitangueira. Ciência Rural, Santa Maria, v. 40, n. 10, p. 2086-2091, 2010. Disponível em: http://www.scielo.br/scielo. php?script=sci_arttext\&pid=S0103-84782010001000006\&lng=en\&nrm=iso. Acesso em: 29 Sept. 2015. Epub Oct 08, 2010. http://dx.doi.org/10.1590/S0103-84782010005000163.

FALCONER, D.S. Introduction to quantitative genetics. Viçosa: UFV, 1987.279 p (in Portuguese).

FALCONER, D. S. Introduction of quantitatives genetics. Longman Group, New York. 1981. $340 \mathrm{p}$. 
FARIAS-NETO, J. T.; YOKOMIZO, G.; BIANCHETTI, A. Coeficientes de repetibilidade genética de caracteres em pupunheira. Revista Brasileira de Fruticultura, Jaboticabal, v. 24, n. 3, p. 731733, 2002.

GONÇALVES, P.S. de; ROSSETTI, A.G.; PAIVA, J.R. de. Coeficiente de repetibilidade e eficiência do miniteste de produção na seleção de plantas de seringueira. Pesquisa Agropecuária Brasileira, Brasília, v. 17, n. 2, p.233-237, 1982.

HOMMA, A. K. O. A extração de recursos naturais renováveis: o caso do extrativismo vegetal na Amazônia. 1989. 575p. Tese (Doutorado em economia aplicada) - Universidade Federal de Viçosa, 1989.

KAINER, K. A. et al. Moist storage of Brazil nut seeds for improved germination and nursery management. Forest Ecology and Management, v. 116, p. 207-217, 1999.

LOCATELLI, M.; SOUZA, V. F. de. Castanha-do-Brasil: características agronômicas, produção de mudas e propagação vegetativa. Porto Velho: EMBRAPA, UEPAE, 1990. 11p. (Circular Técnica, 17).

LOCATElli, M. et al. Cultivo da Castanha do Brasil em Rondônia, Sistemas de Produção, 7, ISSN 1807-1805 Versão Eletrônica, 2005. Disponível em: http://sistemasdeproducao.cnptia. embrapa.br/FontesHTML/Castanha/CultivodaCastanhadoBrasilRO/autores.htm. Acesso em: $28 / 09 / 2015$.

LOPES, R. et al. Repetibilidade de características do fruto de aceroleira. Pesquisa Agropecuária Brasileira, Brasília, v. 36, n. 3, p. 507-513, 2001.

MOREIRA, P. Recomendações técnicas para formação de mudas de castanha-do-brasil (Bertholletia excelsa HBK). Rio Branco: EMBRAPA - CPAF Acre, 1993. 25 p. (EMBRAPA-CPAF Acre. Documentos, 18)

MORITZ, A. Estudos biológicos da floração e frutificação da Castanha-do-brasil (Bertholletia excelsa H.B.K). EMBRAPA. Belém, Brasil. 1984.

MULAMBA, N.N.; MOCK, J.J. Improvement of yield potential of the Eto Blanco maize (Zea mays L.) population by breeding for plant traits. Egypt Journal of Genetics and Cytology, v.7, n.1, p.40-51, 1978.

MÜlleR, C.H.; CAlZAVARA, B.B.G. Castanha-do-Brasil. Recomendações Básicas 11. Embrapa, Centro de Pesquisa Agropecuária do Trópico Úmido, 1989. 6 p.

MÜlleR, C. H. et al. B. A cultura da castanha-do-brasil. Empresa Brasileira de Pesquisa Agropecuária, centro de Pesquisa Agroflorestal da Amazônia Oriental, Coleção Plantar, 23, 65p. 1995.

NASCIMENTO, W. et al. Castanha-do-Brasil (Bertholletia excelsa H. B.). Serviço Brasileiro Florestal, SBF. Brasília. 61p. 2010.

NEGREIROS, J. R. DA S. et al. Estimativas de repetibilidade de caracteres de produção em laranjeiras-doces no Acre. Pesquisa Agropecuária Brasileira, Brasília, v.43, n.12, pp. 1763-1768, 2008.

O’MALLEY, D. M. et al. Genetics of Brazil nut (Bertholletia excelsa Humb. \& Bonpl. Lecythidaceae), 2. Mating system. Theorical Apply Genetic, v.76, p.929- 932, 1988.

PEDROZO, C. A. et al. Repeatability of fruits and seeds production and selection of brazil nut genotypes in native populations in Roraima. Revista Árvore, Viçosa, v. 39, n. 5, p. 863-871, 2015.

PEREIRA, A. V. et al. Influência da estabilização de genótipos de capim elefante (Pennisetum purpureum Jacq). Revista Brasileira de Zootecnia, Viçosa, v.26, n.4, p.762-767, 2002. 
RESENDE, M.D.V. Software Selegen - REML/BLUP. Documentos Embrapa Florestas, Colombo, n.77, 2006.

RESENDE, M.D.V. Genética biométrica e estatística no melhoramento de plantas perenes. Brasília: Embrapa Informação Tecnológica, 2002.

RUIZ, R. C. Análise Preliminar das Cadeias da Borracha e da Castanha, em nível Nacional e Identificação de Territórios Estratégicos. Ministério do Meio Ambiente, Secretaria de Políticas para o Desenvolvimento Sustentável. Relatório BRA 99/025 - Produto 2. 43p. 2008.

SIQUEIRA. E. R. Coeficiente de repetibilidade da produção de frutos do coqueiro comum. Pesquisa Agropecuária Brasileira, Brasília, v. 17, n. 4, p. 573-574, 1982.

STOIAN, D. Cosechando lo que cae: la economía de la castaña Bertholletia excelsa H.B.K em la amazônia boliviana. In: ALEXIADES, M.N.; SHANLEY, P. (Eds.). Productos forestales, medios de subsistencia y conservación de productos forestales no maderables. Borgor: Cifor, v.3, p.89116, 2004.

SUJII, P. S. et al. Genetic structure of Bertholletia excelsa populations from the Amazon at different spatial scales. Conservation Genetics, v. 16, p. 955-964, 2015.

VASCONCELOS, M. E. C. et al. Métodos de estimação do coeficiente de repetibilidade da seringueira. Pesquisa Agropecuária Brasileira, Brasília, v. 20, n. 4, p. 433-437, 1985.

WADT, L. H. O. et al. Sustainable forest use in Brazilian extractive reserves: Natural regeneration of Brazil nut in exploited populations. Biological Conservation, v.141, p.332-346, 2008.

WADT, L.H.O.; KAINER, K.A. Domesticação e melhoramento da castanheira. In: BORÉM, M. T. G. L.; CHARLES, R. C (Eds.) Domesticação e melhoramento: espécies amazônicas. Viçosa, MG, 2009. 